Research Article

\title{
Toxicokinetics of 11 Gelsemium Alkaloids in Rats by UPLC- MS/MS
}

\author{
Xiuwei Shen $\mathbb{D}$, ${ }^{1}$ Jianshe $M a \mathbb{D}^{2},{ }^{2}$ Xianqin Wang $\mathbb{D}^{3}$, Congcong Wen $\mathbb{D}{ }^{4}$ \\ and Meiling Zhang ${ }^{3}{ }^{3}$ \\ ${ }^{1}$ Ruian People's Hospital, The Third Affiliated Hospital of Wenzhou Medical University, Wenzhou 325000, China \\ ${ }^{2}$ School of Basic Medicine, Wenzhou Medical University, Wenzhou 325035, China \\ ${ }^{3}$ Analytical and Testing Center, School of Pharmaceutical Sciences, Wenzhou Medical University, Wenzhou 325035, China \\ ${ }^{4}$ Laboratory Animal Center of Wenzhou Medical University, Wenzhou 325035, China
}

Correspondence should be addressed to Meiling Zhang; zml@wmu.edu.cn

Received 22 April 2020; Revised 1 June 2020; Accepted 16 June 2020; Published 15 July 2020

Academic Editor: Damião Pergentino de Sousa

Copyright (C) 2020 Xiuwei Shen et al. This is an open access article distributed under the Creative Commons Attribution License, which permits unrestricted use, distribution, and reproduction in any medium, provided the original work is properly cited.

Gelsemium elegans (Gardn. \& Champ.) Benth. is a plant belonging to the genus Gelsemium (family Gelsemiaceae), and its main components are alkaloids. It is a Chinese traditional medicinal plant and notoriously known as a highly toxic medicine. However, a method has not yet been found for the simultaneous detection of 11 Gelsemium alkaloids in rat plasma, and the toxicokinetics of 11 Gelsemium alkaloids after intravenous administration has not been reported. In this work, we have developed a sensitive and rapid method of ultraperformance liquid chromatography/tandem mass spectrometry (UPLC-MS/MS) for the detection of 11 Gelsemium alkaloids in rat plasma. The toxicokinetic behavior was also investigated, so as to provide a reference of the scientific properties of Gelsemium elegans and improve the efficacy and safety of drugs. Sixty-six Sprague-Dawley rats were randomly divided into 11 groups, six rats in each group. Each group was intravenously given one alkaloid (0.1 mg/kg), respectively. A Waters UPLC BEH C18 column $(50 \mathrm{~mm} \times 2.1 \mathrm{~mm}, 1.7 \mu \mathrm{m})$ was used for chromatographic separation. Methanol and water (containing $0.1 \%$ formic acid) were used for the mobile phase with gradient elution. Multiple reactions were monitored, and positive electrospray ionization was used for quantitative analysis. The precision was less than $16 \%$, and the accuracy was between $86.9 \%$ and $113.2 \%$. The extraction efficiency was better than $75.8 \%$, and the matrix effects ranged from $88.5 \%$ to $107.8 \%$. The calibration curves were in the range of $0.1-200 \mathrm{ng} / \mathrm{mL}$, with a correlation coefficient $\left(R^{2}\right)$ greater than 0.995 . The UPLC-MS/MS method was successfully applied to the toxicokinetics of 11 Gelsemium alkaloids in rats after intravenous administration $(0.1 \mathrm{mg} / \mathrm{kg}$ for each alkaloid). The results of the toxicokinetics provide a basis for the pharmacology and toxicology of Gelsemium alkaloids and scientific evidence for the clinical use of Gelsemium alkaloids.

\section{Introduction}

About 12,000 kinds of alkaloids have been found, most of which have relatively strong physiological activity. Many alkaloids have been widely used in the field of medicine [1]. For example, morphine plays an analgesic role in opium [2]. Codeine, also an alkaloid, can relieve a cough, and ephedrine plays an antiasthmatic role in ephedra [3]. Chinese herbal medicines often contain poisonous alkaloids, which have the functions of antitumor, antivirus, antiplatelet aggregation, anti-inflammatory, antiarrhythmia, and antihyper- tension. These officinal values have attracted great attention from the international medical and pharmaceutical circles [4]. Gelsemium elegans (Gardn. \& Champ.) Benth. is a plant belonging to the genus Gelsemium (family Gelsemiaceae), whose main components are alkaloids. It is warm, pungent, bitter, and poisonous. It is mainly distributed in the Fujian and Zhejiang provinces in China. It is a Chinese traditional medicinal plant and also a world-famous highly toxic medicine [5]. In China, it is mainly used for external application because of its high toxicity. It can dispel wind and blood stasis, reduce swelling, relieve pain, and kill insects. In recent 
years, pharmacological studies have shown that Gelsemium elegans has many functions, including analgesic effects, sedation, anti-inflammation, mydriatic effects, antitumor, reduced heart rate, heart contraction inhibition, increasing blood pressure, and improving immunity $[6,7]$. The antitumor effect of Gelsemium elegans provides an attractive prospect for research and development. Indole alkaloids are the main chemical components in Gelsemium elegans and the main source of its toxicity. However, as the toxic dose is close to the effective dose, poisoning events of the Gelsemium elegans are common in clinical practice. Seventeen kinds of poisonous alkaloids have been isolated from Gelsemium elegans, among which koumine is the most abundant, followed by gelsemine. As the alkaloid types and contents from different places and different plant parts of Gelsemium elegans are slightly different, there are currently not many reports regarding the toxicokinetics of Gelsemium alkaloids in vivo [8-10]. It has been a popular research topic to analyze alkaloids from different sources in complex systems quickly, sensitively, and reliably. UPLC-MS/MS, because of its flexibility, exhibits advantages of good sensitivity, excellent separation ability, wide application range, and strong specificity, which is why it is widely used in alkaloid analysis [11-14].

There have been methods reported for the determination of Gelsemium alkaloids, including high performance liquid chromatography-tandem (HPLC) $[15,16]$, ultraperformance liquid chromatography-quadrupole-time of flight mass spectrometry (UPLC-Q-TOF/MS) [17, 18], liquid chromatography/tandem mass spectrometry (LC/MS/MS) [19-23], and ultraperformance liquid chromatography/tandem mass spectrometry (UPLC-MS/MS) [24-28] in vivo. However, a method has not been found for the simultaneous determination of 11 Gelsemium alkaloids in rat plasma, and the toxicokinetics of 11 Gelsemium alkaloids (humantenirine, humantenine, akuammidine, gelsevirine, rankinidine, n-methoxyanhydrovobasinediol, gelsenicine, gelsemine, koumine, koumidine, and sempervirine) after intravenous administration has not been reported. In this work, a sensitive and rapid method of UPLC-MS/MS was developed for the determination of 11 Gelsemium alkaloids in rat plasma, and the toxicokinetic behavior was investigated, so as to provide a scientific understanding of Gelsemium elegans and improve the efficacy and safety of drugs.

\section{Materials and Methods}

2.1. Materials and Reagents. Humantenirine, humantenine, akuammidine, gelsevirine, rankinidine, $n$-methoxyanhydrovobasinediol, gelsenicine, gelsemine, koumine, koumidine, sempervirine (all $>98 \%$, Figure 1), and the internal standard strychnine (IS, all $>98 \%$ ) were purchased from Chengdu Mansite Biotechnology Co., Ltd (Chengdu, China). HPLC grade methanol and acetonitrile were obtained from Merck Company (Darmstadt, Germany). Ultrapure water (resistance $>18 \mathrm{~m} \Omega$ ) was prepared by Millipore Milli-Q (Bedford, USA).

2.2. Instrumentation and Conditions. An ACQUITY H-Class UPLC and XEVO TQ-S micro triple quadrupole mass spectrometer was obtained from Waters Corp. (Milford, MA, USA).

Eleven Gelsemium alkaloids (humantenirine, humantenine, akuammidine, gelsevirine, rankinidine, n-methoxyanhydrovobasinediol, gelsenicine, gelsemine, koumine, koumidine, and sempervirine) and IS were separated using a Waters $\mathrm{UPLC}^{\circledR} \mathrm{BEH} \mathrm{C18}$ column $(50 \mathrm{~mm} \times 2.1 \mathrm{~mm}, 1.7 \mu \mathrm{m})$ maintained at $40^{\circ} \mathrm{C}$. The mobile phase consisted of methanol and water (containing $0.1 \%$ formic acid). The gradient elution was as follows: from 0 to $0.2 \mathrm{~min}$, the methanol was kept at $10 \%$; from 0.2 to $2.0 \mathrm{~min}$, methanol increased from $10 \%$ to $80 \%$; from 2.0 to $2.5 \mathrm{~min}$, the methanol was kept at $80 \%$; from 2.5 to $2.8 \mathrm{~min}$, methanol was changed from $80 \%$ to $10 \%$; and at last, methanol was kept at $10 \%$ for $2.2 \mathrm{~min}$. The flow rate was set at $0.4 \mathrm{~mL} / \mathrm{min}$.

Nitrogen was used for the cone gas $(50 \mathrm{~L} / \mathrm{h})$ and desolvation gas $(900 \mathrm{~L} / \mathrm{h})$. The capillary voltage was $2.5 \mathrm{kV}$, the source temperature was $150^{\circ} \mathrm{C}$, and the desolvation temperature was $450^{\circ} \mathrm{C}$. The employed collision gas for fragmentation in the multiple reaction monitoring (MRM) mode was argon. The MRM mode (Table 1) was used for quantitative analysis in the electrospray ionization (ESI) positive interface (Figure 2).

2.3. Stock Solutions. Stock solutions of $100 \mu \mathrm{g} / \mathrm{mL}$ each of humantenirine, humantenine, akuammidine, gelsevirine, rankinidine, n-methoxyanhydrovobasinediol, gelsenicine, gelsemine, koumine, koumidine, sempervirine, and IS were prepared in methanol. The working standard solutions of each of the 11 Gelsemium alkaloids were prepared by dilution of the stock solution with methanol. The stock solutions and working standard solutions were stored at $4^{\circ} \mathrm{C}$.

The calibration standards were prepared by spiking blank rat plasma with appropriate amounts of the 11 Gelsemium alkaloid working standard solutions. Calibration plots of each of the 11 Gelsemium alkaloids were constructed in the $0.1-200 \mathrm{ng} / \mathrm{mL}$ range for plasma $(0.1,0.5,2,10,20,50,100$, and $200 \mathrm{ng} / \mathrm{mL}$ ). Quality-control (QC) samples $(0.4,18$, and $180 \mathrm{ng} / \mathrm{mL}$ ) were prepared in the same manner as the calibration standards.

2.4. Sample Preparation. In a $1.5 \mathrm{~mL}$ centrifuge tube, an aliquot of $50 \mu \mathrm{L}$ plasma was added; then, $150 \mu \mathrm{L}$ acetonitrile (containing IS $20 \mathrm{ng} / \mathrm{mL}$ ) was added, and the vortex was mixed for $1.0 \mathrm{~min}$, then centrifuged $(14900 \mathrm{~g})$ for $10 \mathrm{~min}$, and then, the supernatant $(2 \mu \mathrm{L})$ was injected into the UPLC-MS/MS system for analysis.

2.5. Method Validation. Calibration curves were established by analyzing calibration samples on three different days. The relationship between the peak area ratio of 11 alkaloids and the concentration of the analyte was fitted to the equations. In the concentration range of $0.1-200 \mathrm{ng} / \mathrm{mL}$, linear regression was performed using the weighting factor $(1 / x)$ of the inverse concentration. LLOQ was calculated as the baseline of blank plasma plus the concentrations of 11 Gelsemium alkaloids added, and the final deviation should be within $\pm 20 \%$. 


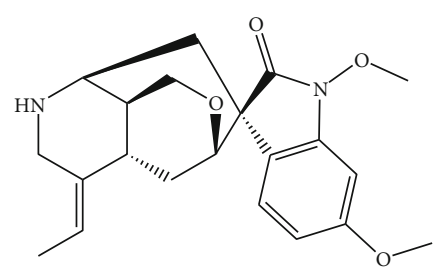

(a)

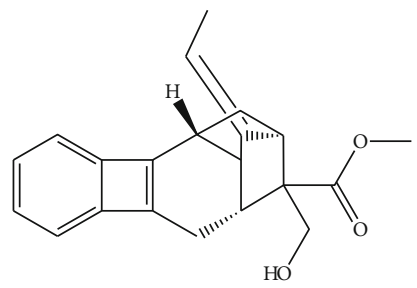

(c)<smiles>C/C=C1\NC2CC3CC(C2)[C@]1(C(=O)N(OC)c1ccccc1)C3</smiles>

(e)

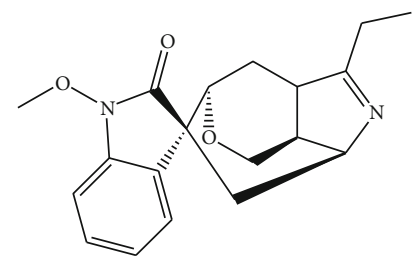

(g)

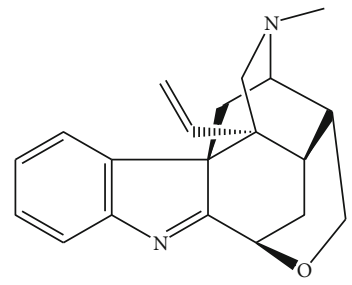

(i)<smiles></smiles>

$(\mathrm{k})$<smiles>C/C=C1/CN2C[C@H]3c4[nH]c5ccccc5c4C[C@H]2N3C(=O)COC1CO</smiles>

(b)

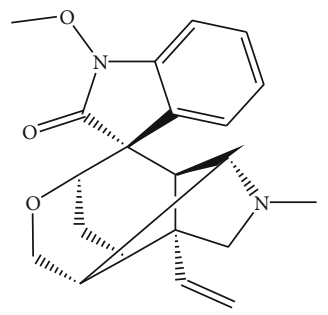

(d)<smiles>CC[C@H]1CN(C)C2CC3c4ccccc4N(OC)C3C3C[C@@H]1[C@H]2CO3</smiles>

(f)

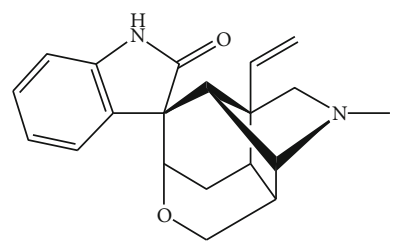

(h)<smiles>C/C=C1\CN2Nc3ccccc3C[C@@H]2[C@H]1CO</smiles>

(j)

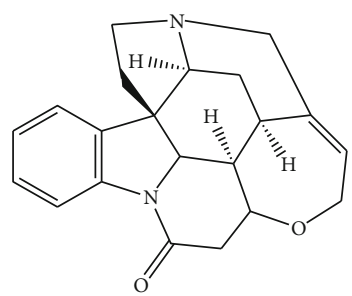

(1)

Figure 1: Chemical structures of humantenirine (a), humantenine (b), akuammidine (c), gelsevirine (d), rankinidine (e), nmethoxyanhydrovobasinediol (f), gelsenicine (g), gelsemine (h), koumine (i), koumidine (j), sempervirine (k), and strychnine (IS, l). 
TABLE 1: Mass parameters for 11 Gelsemium alkaloids and strychnine (IS).

\begin{tabular}{|c|c|c|c|c|}
\hline Compound name & Parent $(m / z)$ & Daughter $(m / z)$ & Cone $(\mathrm{V})$ & Collision (V) \\
\hline Humantenirine & 371.2 & 340.1 & 38 & 14 \\
\hline Humantenine & 355.2 & 324.2 & 24 & 16 \\
\hline Akuammidine & 353.1 & 166.1 & 28 & 26 \\
\hline Gelsevirine & 353.1 & 322.1 & 22 & 40 \\
\hline Rankinidine & 341.1 & 310.1 & 14 & 20 \\
\hline n-Methoxyanhydrovobasinediol & 339.2 & 308.1 & 12 & 14 \\
\hline Gelsenicine & 327.1 & 296.1 & 36 & 15 \\
\hline Gelsemine & 323.1 & 236.1 & 46 & 22 \\
\hline Koumine & 307.1 & 219.9 & 52 & 38 \\
\hline Koumidine & 295.1 & 143.9 & 24 & 25 \\
\hline Sempervirine & 273.2 & 257.0 & 24 & 25 \\
\hline Strychnine (IS) & 335.2 & 184.2 & 25 & 22 \\
\hline
\end{tabular}

Precision and accuracy were assessed by measuring six replicate QC samples within three days of validation. Precision was expressed by the coefficient of variation $(\mathrm{CV})$. Accuracy was determined as the extent to which the mean value corresponded to the true value.

The selectivity of this method was evaluated by analyzing six batches of blank rat plasma, blank plasma supplemented with 11 alkaloids and IS, and one rat plasma sample.

To evaluate matrix effects, blank rat plasma was extracted, and then, $0.4,18$, and $180 \mathrm{ng} / \mathrm{mL}$ of analyte were added. Then, the corresponding peak area was compared to the peak area of the pure standard solution at the same concentration, and this peak area ratio was defined as the matrix effect.

The extraction efficiency was evaluated by comparing the peak area of the extracted QC sample with the peak area $(n=6)$ of the reconstituted reference QC solution in the blank plasma extract.

The stability of 11 alkaloids in rat plasma was evaluated by analyzing three replicate plasma samples at $0.4,18$, and $180 \mathrm{ng} / \mathrm{mL}$ under different conditions [29]. The short-term stabilities of the samples after exposure were determined at room temperature for $2 \mathrm{~h}$, and injection samples (after protein precipitation) were performed by UPLC at room temperature for $12 \mathrm{~h}$. Three complete freeze/thaw $(-20$ to $25^{\circ} \mathrm{C}$ ) cycles were evaluated on consecutive days after freezing/thawing stabilization. After storage at $-20^{\circ} \mathrm{C}$ for 30 days, its long-term stability was evaluated.

2.6. Toxicokinetics. Sixty-six Sprague Dawley rats (male, 200-220 g) obtained from the Laboratory Animal Center of Wenzhou Medical University were randomly divided into 11 groups, six rats for each group. All experimental procedures and operating procedures were reviewed and approved by the Animal Care and Use Committee of Wenzhou Medical University. Each group was intravenously given humantenirine $(0.1 \mathrm{mg} / \mathrm{kg})$, humantenine $(0.1 \mathrm{mg} / \mathrm{kg})$, akuammidine $(0.1 \mathrm{mg} / \mathrm{kg})$, gelsevirine $(0.1 \mathrm{mg} / \mathrm{kg})$, rankinidine $(0.1 \mathrm{mg} / \mathrm{kg})$, n-methoxyanhydrovobasinediol $(0.1 \mathrm{mg} / \mathrm{kg})$, gelsenicine $(0.1 \mathrm{mg} / \mathrm{kg})$, gelsemine $(0.1 \mathrm{mg} / \mathrm{kg})$, koumine $(0.1 \mathrm{mg} / \mathrm{kg})$, koumidine $(0.1 \mathrm{mg} / \mathrm{kg})$, and sempervirine $(0.1 \mathrm{mg} / \mathrm{kg})$, respectively.
Blood samples $(0.3 \mathrm{~mL})$ were collected from the tail vein into heparinized tubes at $0.0833,0.5,1,2,3,4,6,8,12$, and $24 \mathrm{~h}$ after intravenous administration and centrifuged for $10 \mathrm{~min}$ at $3000 \mathrm{~g}$, and $100 \mu \mathrm{L}$ plasma was obtained. Toxicokinetics was analyzed by DAS software (Version 3.0, China Pharmaceutical University).

\section{Results}

3.1. Method Validation. Equations of the calibration curves (0.1-200 ng/mL) are listed in Table 2. The LLOQ for Gelsemium alkaloids were $0.1 \mathrm{ng} / \mathrm{mL}$.

Intraday and interday precision was measured to be $16 \%$ or less. The accuracy ranged from $86.9 \%$ to $113.2 \%$. Extraction efficiency was between $75.8 \%$ and $98.4 \%$ (Table 3 ).

Figure 3 shows the typical UPLC-MS/MS of blank rat plasma spiked with 11 Gelsemium alkaloids and IS. No interfering endogenous substances were observed at the retention time of the 11 Gelsemium alkaloids and IS.

The matrix effects were measured to be $88.5-107.8 \%$ (Table 3), indicating that matrix effects from plasma are negligible.

The stability results indicate that the 11 Gelsemium alkaloids are stable under the three storage conditions since the precision was within $\pm 15 \%$ (Table 4 ).

3.2. Toxicokinetics. The UPLC-MS/MS method was applied to the toxicokinetic study of 11 Gelsemium alkaloids in rats. The main toxicokinetic parameters after intravenous administration of humantenirine, humantenine, akuammidine, gelsevirine, rankinidine, n-methoxyanhydrovobasinediol, gelsenicine, gelsemine, koumine, koumidine, and sempervirine are summarized in Table 5, using noncompartment model analysis. The 11 Gelsemium alkaloid plasma mean concentration-time curves are shown in Figure 4.

\section{Discussion}

The feasibility of ESI in negative and positive ion modes was evaluated in this work. ESI is suitable for compounds with medium polarity to high polarity. APCI has the advantage 


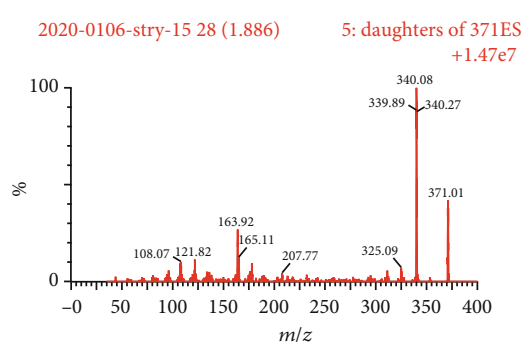

(a)



(c)

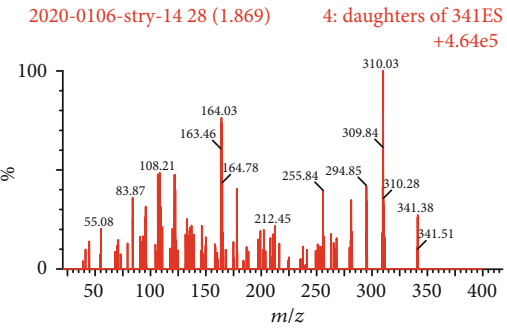

(e)



(g)

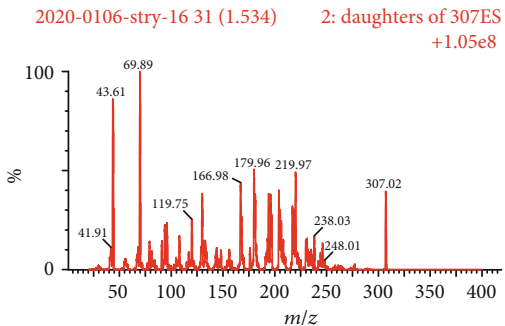

(i)

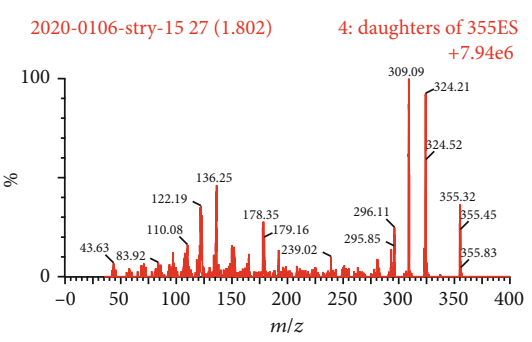

(b)

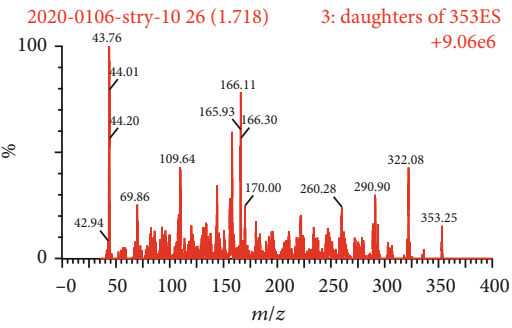

(d)



(f)

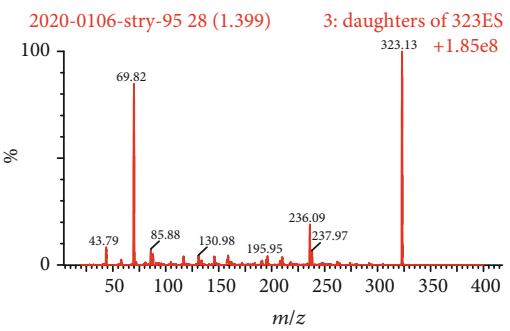

(h)

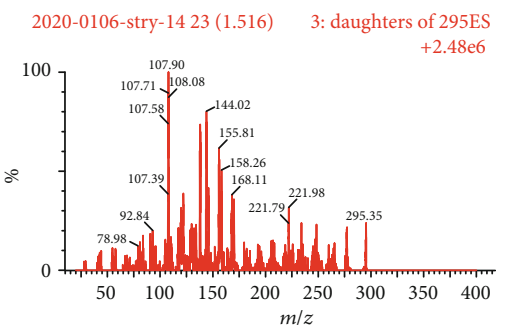

(j)

Figure 2: Continued. 


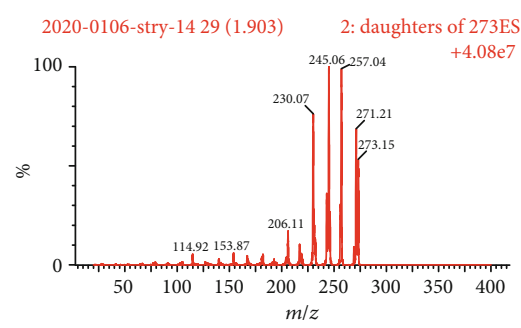

(k)

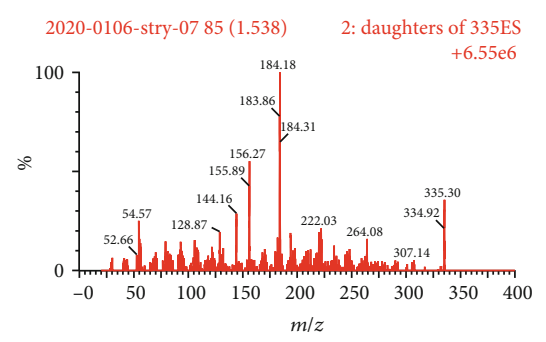

(1)

Figure 2: Mass spectra of humantenirine (a), humantenine (b), akuammidine (c), gelsevirine (d), rankinidine (e), n-methoxyanhydrovobasinediol (f), gelsenicine (g), gelsemine (h), koumine (i), koumidine (j), sempervirine (k), and strychnine (IS, l).

TABLE 2: Regression equations and correlation coefficients for 11 Gelsemium alkaloids in rat plasma.

\begin{tabular}{lccc}
\hline Compound & Linear range $(\mathrm{ng} / \mathrm{mL})$ & Regression equation & Correlation coefficient \\
\hline Humantenirine & $0.1-200$ & $y=0.0125 x-0.0041$ & 0.9962 \\
Humantenine & $0.1-200$ & $y=0.0092 x-0.0053$ & 0.9991 \\
Akuammidine & $0.1-200$ & $y=0.0058 x+0.0134$ & 0.9992 \\
Gelsevirine & $0.1-200$ & $y=0.0136 x-0.0060$ & 0.9995 \\
Rankinidine & $0.1-200$ & $y=0.0117 x+0.0067$ & 0.9987 \\
n-Methoxyanhydrovobasinediol & $0.1-200$ & $y=0.0153 x+0.0125$ & 0.9993 \\
Gelsenicine & $0.1-200$ & $y=0.0161 x+0.0010$ & 0.9985 \\
Gelsemine & $0.1-200$ & $y=0.0018 x-0.0013$ & 0.9967 \\
Koumine & $0.1-200$ & $y=0.0043 x-0.0014$ & 0.9980 \\
Koumidine & $0.1-200$ & $y=0.0035 x+0.0025$ & 0.9998 \\
Sempervirine & $0.1-200$ & $y=0.0048 x+0.0028$ & 0.9950 \\
\hline
\end{tabular}

$y=$ peak area ratio of Gelsemium alkaloids versus IS; $x$ = concentration of Gelsemium alkaloids.

of weak polarity to medium polarity. ESI is more suitable for the analysis of alkaloids. The sample structure contains complex nitrogen-containing heterocycles, so the positive mode should be preferred [30-32]. When the positive and negative modes were used to monitor the alkaloids simultaneously, the characteristic molecular ion peaks of 11 Gelsemium alkaloids had low sensitivity in the negative mode. There, ESI with the positive ion mode was used for detection of 11 Gelsemium alkaloids. The optimum conditions of mass spectrometric analysis of alkaloids were investigated. Through the study of spray needle voltage, drying gas temperature, dry gas pressure, capillary voltage, and impact energy, the best mass spectrometric conditions were obtained and are shown in Figure 2.

The conditions of the chromatographic separation of alkaloids were investigated. Through the study of the mobile phase, stationary phase, flow rate, and column temperature, the separation conditions were optimized. $0.1 \%$ formic acid was added into the mobile phase, and the alkaloids responded well, because the alkaloids favor ionization under the positive ion mode within acidic conditions. The mobile phase of methanol-0.1\% formic acid using gradient elution almost achieved baseline separation for humantenirine, humantenine, akuammidine, gelsevirine, rankinidine, n-methoxyanhydrovobasinediol, gelsenicine, gelsemine, koumine, koumidine, and sempervirine. However, some peaks did not achieve baseline separation, and the MRM mode was used for quantitative analysis in our work, where the quantitative ion pairs were different.

Several compounds including strychnine, carbamazepine, diazepam, midazolam, and berberine were tested for IS. Strychnine was chosen as IS because of its similar mass ionization and retention time as the 11 Gelsemium alkaloids in a positive-ion ESI mode. Sample treatment prior to UPLC-MS/MS analysis was very important $[33,34]$. An efficient and simple method of protein precipitation was used in our work. Choosing acetonitrile as the protein precipitation solvent can get better extraction efficiency and matrix effects than methanol.

The developed UPLC-MS/MS was used for the subsequent quantification of all Gelsemium alkaloids, which has a faster analysis time than traditional HPLC $[35,36]$ or LC-MS [37-39] and greatly enhances the signal intensity. It only takes five minutes to analyze a plasma sample, which can save significant time when analyzing hundreds of samples. In addition, the LLOQ of the 11 Gelsemium alkaloids was relatively low $(0.1 \mathrm{ng} / \mathrm{mL})$. LLOQ was calculated as the baseline of blank plasma plus the concentrations of the 11 Gelsemium alkaloids added, and the final deviation was within $\pm 20 \%$. 
TABle 3: Accuracy, precision, extraction efficiency, and matrix effects of 11 Gelsemium alkaloids in rat plasma $(n=6)$.

\begin{tabular}{|c|c|c|c|c|c|c|c|}
\hline \multirow{2}{*}{ Compound } & \multirow{2}{*}{ Concentration $(\mathrm{ng} / \mathrm{mL})$} & \multicolumn{2}{|c|}{ Accuracy (\%) } & \multicolumn{2}{|c|}{ CV (\%) } & \multirow{2}{*}{ Matrix effect (\%) } & \multirow{2}{*}{ Recovery (\%) } \\
\hline & & Intraday & Interday & Intraday & Interday & & \\
\hline \multirow{4}{*}{ Humantenirine } & 0.1 & 103.2 & 98.4 & 5.7 & 9.9 & 95.4 & 84.0 \\
\hline & 0.4 & 95.5 & 95.4 & 7.1 & 13.7 & 93.6 & 81.8 \\
\hline & 18 & 99.4 & 99.2 & 3.0 & 7.5 & 97.3 & 89.9 \\
\hline & 180 & 99.5 & 107.4 & 7.7 & 8.8 & 93.6 & 86.8 \\
\hline \multirow{4}{*}{ Humantenine } & 0.1 & 98.4 & 104.7 & 7.3 & 13.0 & 99.1 & 85.8 \\
\hline & 0.4 & 97.7 & 98.3 & 2.1 & 5.2 & 98.8 & 89.1 \\
\hline & 18 & 102.0 & 100.8 & 3.7 & 5.0 & 103.6 & 86.0 \\
\hline & 180 & 96.5 & 106.3 & 4.7 & 8.4 & 98.1 & 90.0 \\
\hline \multirow{4}{*}{ Akuammidine } & 0.1 & 107.9 & 100.8 & 5.9 & 12.7 & 103.4 & 98.4 \\
\hline & 0.4 & 107.3 & 101.7 & 8.0 & 6.7 & 94.1 & 95.4 \\
\hline & 18 & 104.3 & 98.0 & 5.2 & 4.5 & 98.0 & 90.5 \\
\hline & 180 & 96.3 & 99.7 & 5.0 & 4.4 & 107.2 & 98.3 \\
\hline \multirow{4}{*}{ Gelsevirine } & 0.1 & 106.5 & 109.2 & 5.7 & 12.3 & 98.5 & 89.7 \\
\hline & 0.4 & 101.1 & 99.5 & 3.2 & 6.5 & 96.6 & 88.9 \\
\hline & 18 & 99.3 & 95.6 & 5.8 & 9.6 & 99.5 & 93.2 \\
\hline & 180 & 102.2 & 103.8 & 7.0 & 6.4 & 101.3 & 96.6 \\
\hline \multirow{4}{*}{ Rankinidine } & 0.1 & 99.3 & 101.5 & 14.9 & 13.3 & 103.2 & 93.8 \\
\hline & 0.4 & 102.2 & 97.9 & 7.8 & 2.2 & 104.2 & 91.0 \\
\hline & 18 & 103.2 & 105.0 & 8.8 & 12.6 & 107.7 & 86.5 \\
\hline & 180 & 97.4 & 101.6 & 7.1 & 5.8 & 103.2 & 92.0 \\
\hline \multirow{4}{*}{ n-Methoxyanhydrovobasinediol } & 0.1 & 92.7 & 100.9 & 5.0 & 8.3 & 98.8 & 95.9 \\
\hline & 0.4 & 106.0 & 97.9 & 4.0 & 4.5 & 97.7 & 89.0 \\
\hline & 18 & 102.7 & 94.9 & 3.4 & 6.2 & 105.4 & 90.9 \\
\hline & 180 & 97.4 & 103.8 & 2.5 & 4.0 & 103.4 & 90.6 \\
\hline \multirow{4}{*}{ Gelsenicine } & 0.1 & 104.5 & 98.2 & 10.9 & 13.8 & 90.6 & 91.6 \\
\hline & 0.4 & 102.8 & 103.5 & 4.0 & 12.3 & 88.5 & 84.3 \\
\hline & 18 & 105.1 & 97.0 & 3.3 & 5.3 & 95.1 & 82.2 \\
\hline & 180 & 92.5 & 92.2 & 6.9 & 5.4 & 98.9 & 93.5 \\
\hline \multirow{4}{*}{ Gelsemine } & 0.1 & 86.9 & 96.7 & 16.7 & 12.1 & 97.2 & 82.5 \\
\hline & 0.4 & 98.9 & 89.9 & 11.3 & 12.7 & 103.8 & 90.1 \\
\hline & 18 & 106.5 & 91.2 & 13.7 & 8.8 & 107.8 & 86.0 \\
\hline & 180 & 92.8 & 102.2 & 8.2 & 11.2 & 91.2 & 84.9 \\
\hline \multirow{4}{*}{ Koumine } & 0.1 & 113.2 & 91.8 & 6.9 & 15.9 & 94.2 & 95.6 \\
\hline & 0.4 & 108.3 & 99.6 & 11.8 & 8.1 & 92.8 & 83.3 \\
\hline & 18 & 99.6 & 91.7 & 8.3 & 11.2 & 91.6 & 82.5 \\
\hline & 180 & 98.2 & 108.3 & 12.7 & 13.9 & 89.6 & 84.7 \\
\hline \multirow{4}{*}{ Koumidine } & 0.1 & 102.3 & 103.9 & 11.1 & 14.6 & 100.8 & 78.2 \\
\hline & 0.4 & 105.5 & 101.2 & 9.5 & 6.1 & 97.7 & 79.8 \\
\hline & 18 & 97.5 & 105.8 & 10.3 & 10.6 & 100.2 & 76.4 \\
\hline & 180 & 99.1 & 96.6 & 5.7 & 8.9 & 94.0 & 82.7 \\
\hline \multirow{4}{*}{ Sempervirine } & 0.1 & 103.0 & 94.2 & 13.8 & 11.7 & 95.9 & 84.7 \\
\hline & 0.4 & 96.5 & 104.3 & 6.4 & 8.8 & 97.9 & 77.4 \\
\hline & 18 & 103.1 & 97.8 & 5.8 & 4.3 & 98.2 & 75.8 \\
\hline & 180 & 101.0 & 96.7 & 9.7 & 4.3 & 106.0 & 85.6 \\
\hline
\end{tabular}


Blank

$12: 38: 4$

21-Jan-2020

2020-0106-stry-77

MRM of 24 channels ES+

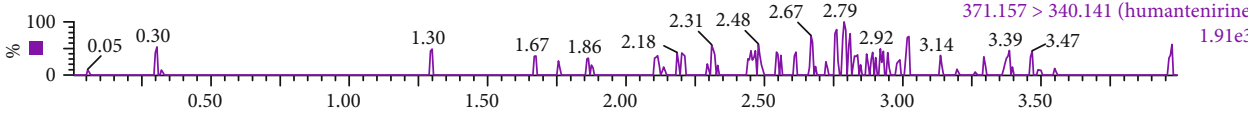

2020-0106-stry-77 $\quad$ MRM of 24 channels ES +

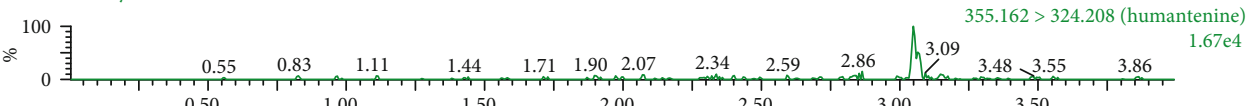

2020-0106-stry-77 $\quad$ MRM of 24 channels ES+

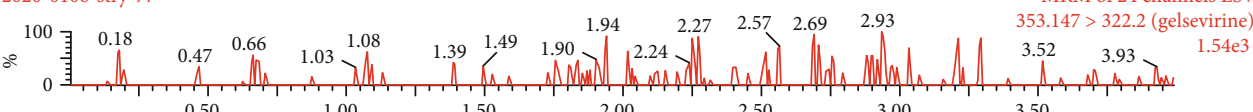

2020-0106-stry-77

MRM of 24 channels ES+

100
0.14

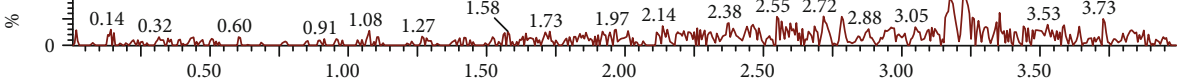

2020-0106-stry-77 $\quad$ MRM of 24 channels ES+

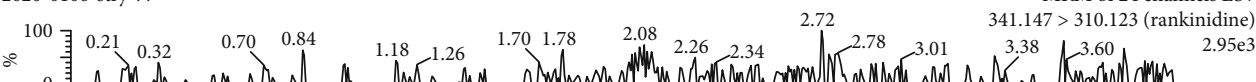

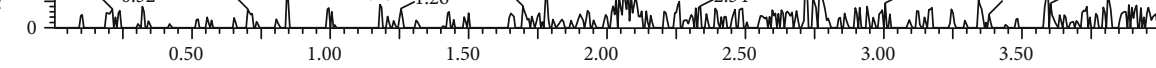

2020-0106-stry-77 MRM of 24 channels ES+

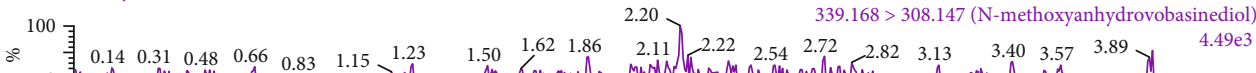

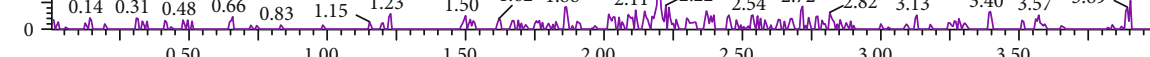

2020-0106-stry-77

$\therefore 00$ 年

2020-0106-stry-77 MRM of 24 channels ES+



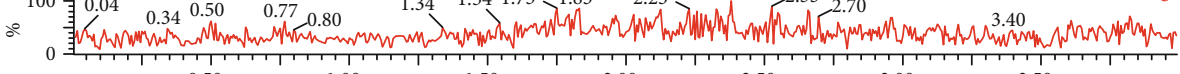

2020-0106-stry-77 MRM of 24 channels ES+

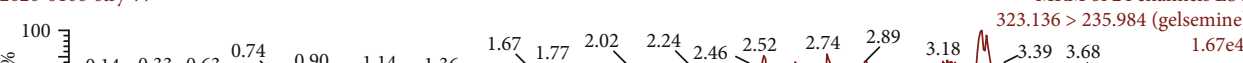

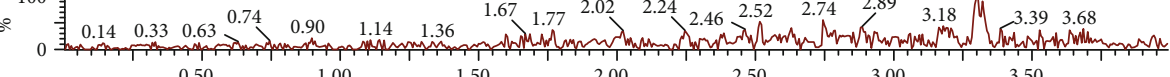

2020-0106-stry-77 $\quad$ MRM of 24 channels ES+

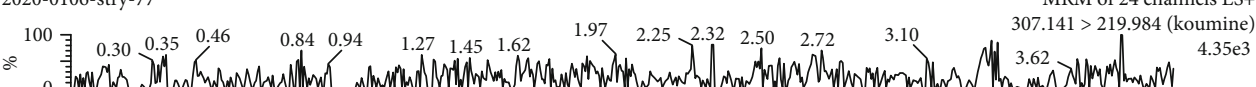

0 :

2020-0106-stry-77 $\quad$ MRM of 24 channels ES

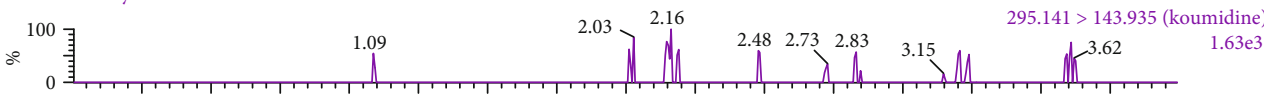

$\begin{array}{llllllll}0.50 & 1.00 & 1.50 & 2.00 & 2.50 & 3.00 & 3.50 \\ \text { MRM of 24 channels ES }+ \\ \text { 2020-0106-stry-77 }\end{array}$

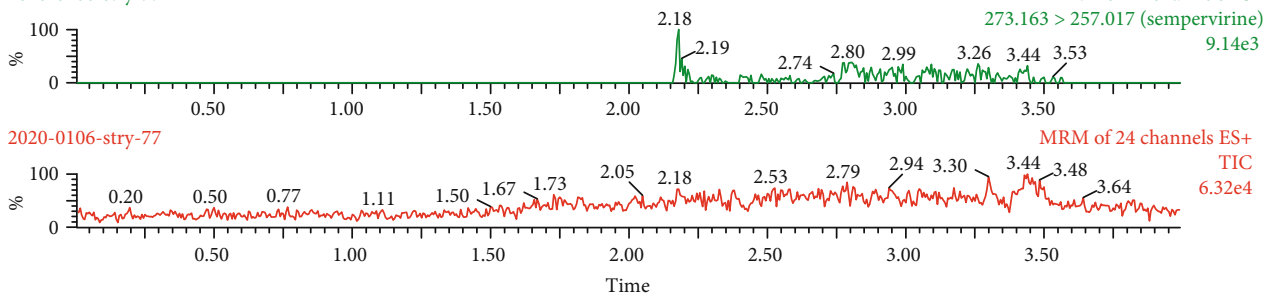

(a)

Figure 3: Continued. 


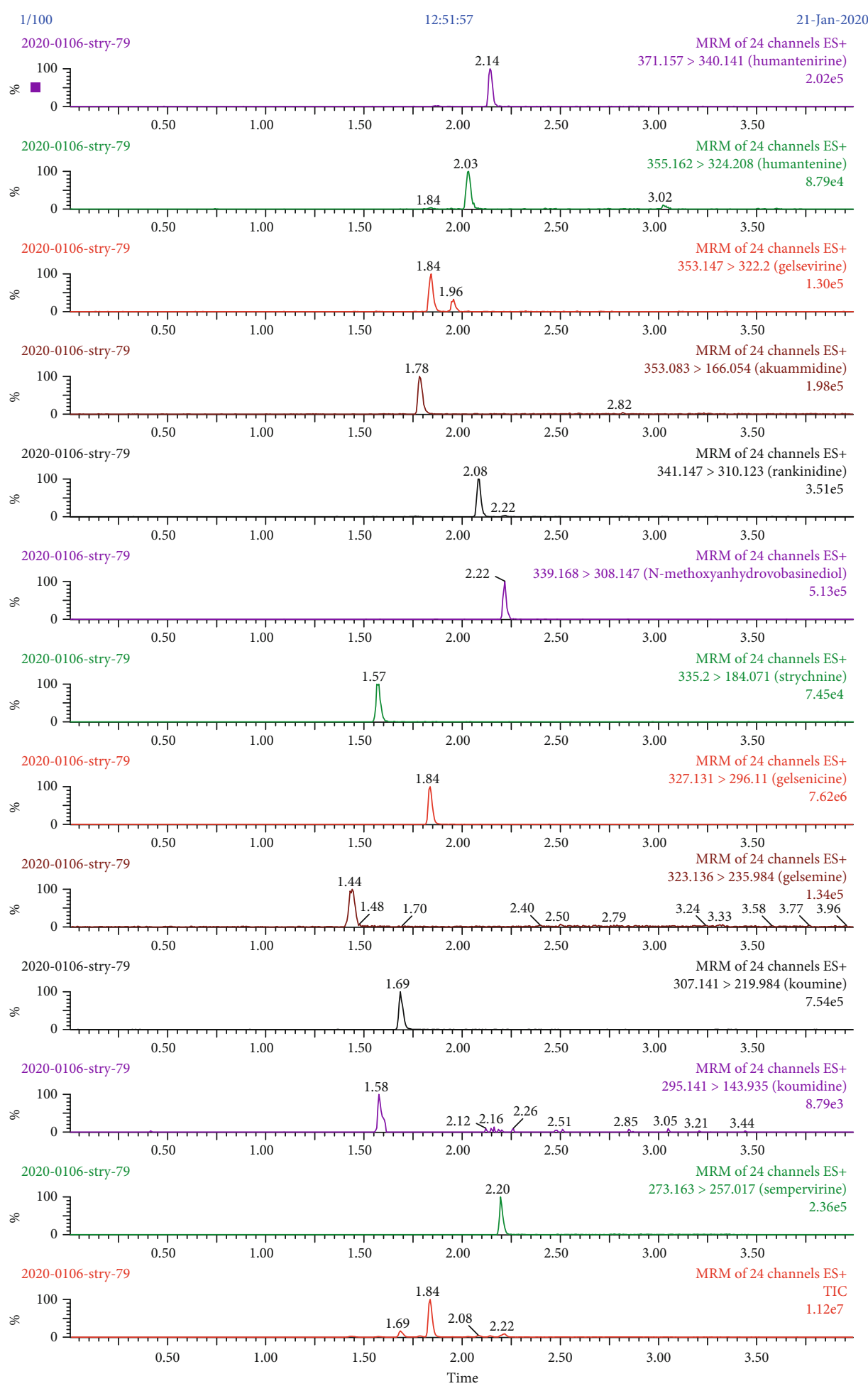

(b)

FIGURE 3: UPLC-MS/MS chromatograms of humantenirine, humantenine, akuammidine, gelsevirine, rankinidine, n-methoxyanhydrovobasinediol, gelsenicine, gelsemine, koumine, koumidine, sempervirine, and strychnine (IS) in rat plasma: (a) blank rat plasma; (b) blank rat plasma spiked with 11 Gelsemium alkaloids and IS. 
TABLE 4: Stability of 11 Gelsemium alkaloids in rat plasma.

\begin{tabular}{|c|c|c|c|c|c|c|c|c|c|}
\hline \multirow{2}{*}{ Compound } & \multirow{2}{*}{$\begin{array}{l}\text { Concentration } \\
(\mathrm{ng} / \mathrm{mL})\end{array}$} & \multicolumn{2}{|c|}{$\begin{array}{c}\text { Autosampler } \\
\text { ambient }\end{array}$} & \multicolumn{2}{|c|}{ Ambient $2 \mathrm{~h}$} & \multicolumn{2}{|c|}{$-20^{\circ} \mathrm{C}$ for $30 \mathrm{~d}$} & \multicolumn{2}{|c|}{ Freeze-thaw } \\
\hline & & $\begin{array}{l}\text { Accuracy } \\
(\%)\end{array}$ & $\begin{array}{l}\mathrm{CV} \\
(\%)\end{array}$ & $\begin{array}{l}\text { Accuracy } \\
(\%)\end{array}$ & $\begin{array}{l}\mathrm{CV} \\
(\%)\end{array}$ & $\begin{array}{c}\text { Accuracy } \\
(\%)\end{array}$ & $\begin{array}{l}\mathrm{CV} \\
(\%)\end{array}$ & $\begin{array}{l}\text { Accuracy } \\
(\%)\end{array}$ & $\begin{array}{l}\mathrm{CV} \\
(\%)\end{array}$ \\
\hline \multirow{3}{*}{ Humantenirine } & 0.4 & 104.2 & 8.5 & 102.1 & 4.0 & 107.9 & 11.7 & 103.0 & 10.5 \\
\hline & 18 & 100.7 & 6.3 & 106.1 & 9.1 & 101.5 & 5.9 & 99.3 & 6.1 \\
\hline & 180 & 98.0 & 5.0 & 106.8 & 7.8 & 99.9 & 5.7 & 97.0 & 8.9 \\
\hline \multirow{3}{*}{ Humantenine } & 0.4 & 94.5 & 5.3 & 98.8 & 7.6 & 107.3 & 12.6 & 99.8 & 11.6 \\
\hline & 18 & 104.4 & 3.6 & 100.8 & 4.6 & 96.5 & 3.5 & 104.0 & 9.6 \\
\hline & 180 & 99.2 & 3.5 & 106.5 & 4.5 & 100.9 & 1.4 & 105.8 & 3.7 \\
\hline \multirow{3}{*}{ Akuammidine } & 0.4 & 102.8 & 7.8 & 98.6 & 8.6 & 111.4 & 11.7 & 90.5 & 14.6 \\
\hline & 18 & 97.6 & 6.7 & 109.7 & 5.9 & 103.5 & 9.6 & 96.8 & 9.4 \\
\hline & 180 & 99.1 & 8.1 & 106.7 & 3.1 & 103.6 & 7.4 & 104.3 & 4.6 \\
\hline \multirow{3}{*}{ Gelsevirine } & 0.4 & 99.5 & 5.4 & 96.9 & 5.8 & 101.3 & 8.5 & 101.1 & 9.2 \\
\hline & 18 & 101.9 & 1.9 & 99.3 & 2.1 & 94.8 & 10.6 & 106.1 & 9.6 \\
\hline & 180 & 96.5 & 4.3 & 103.7 & 4.8 & 93.3 & 10.0 & 99.8 & 7.8 \\
\hline \multirow{3}{*}{ Rankinidine } & 0.4 & 99.5 & 7.8 & 90.3 & 9.1 & 89.7 & 8.5 & 87.4 & 11.7 \\
\hline & 18 & 101.8 & 6.0 & 101.4 & 6.3 & 105.5 & 9.8 & 107.4 & 3.4 \\
\hline & 180 & 96.6 & 6.9 & 106.5 & 5.1 & 104.7 & 7.9 & 101.6 & 5.7 \\
\hline \multirow{3}{*}{ n-Methoxyanhydrovobasinediol } & 0.4 & 96.0 & 8.3 & 94.4 & 6.1 & 102.5 & 4.6 & 92.8 & 9.7 \\
\hline & 18 & 103.8 & 4.3 & 104.1 & 8.5 & 95.3 & 8.9 & 99.8 & 8.3 \\
\hline & 180 & 99.3 & 6.7 & 95.0 & 7.2 & 98.9 & 5.2 & 108.9 & 8.3 \\
\hline \multirow{3}{*}{ Gelsenicine } & 0.4 & 92.4 & 11.7 & 106.6 & 5.3 & 100.8 & 7.3 & 92.1 & 7.4 \\
\hline & 18 & 98.5 & 6.0 & 93.1 & 11.1 & 94.2 & 10.5 & 105.6 & 11.5 \\
\hline & 180 & 105.0 & 9.8 & 103.8 & 12.0 & 92.0 & 5.3 & 104.5 & 13.3 \\
\hline \multirow{3}{*}{ Gelsemine } & 0.4 & 100.8 & 7.4 & 97.2 & 12.6 & 111.4 & 14.0 & 99.7 & 11.5 \\
\hline & 18 & 96.6 & 3.1 & 104.3 & 11.1 & 104.2 & 13.2 & 110.7 & 8.5 \\
\hline & 180 & 95.8 & 2.6 & 104.7 & 10.1 & 103.0 & 8.6 & 91.5 & 3.7 \\
\hline \multirow{3}{*}{ Koumine } & 0.4 & 100.6 & 10.4 & 112.5 & 12.1 & 108.1 & 13.0 & 105.8 & 14.7 \\
\hline & 18 & 97.5 & 8.5 & 108.5 & 7.7 & 88.7 & 11.4 & 104.5 & 14.8 \\
\hline & 180 & 102.3 & 8.7 & 93.7 & 4.7 & 94.1 & 5.4 & 92.5 & 7.4 \\
\hline \multirow{3}{*}{ Koumidine } & 0.4 & 98.6 & 7.5 & 97.2 & 6.2 & 97.5 & 14.8 & 108.1 & 13.0 \\
\hline & 18 & 100.2 & 6.6 & 103.1 & 4.4 & 107.1 & 9.6 & 96.0 & 9.2 \\
\hline & 180 & 104.8 & 5.5 & 101.5 & 6.6 & 92.8 & 3.5 & 101.4 & 7.8 \\
\hline \multirow{3}{*}{ Sempervirine } & 0.4 & 105.3 & 5.4 & 98.3 & 6.9 & 107.8 & 7.5 & 108.5 & 10.5 \\
\hline & 18 & 99.9 & 5.3 & 99.7 & 2.9 & 100.5 & 10.8 & 95.4 & 8.3 \\
\hline & 180 & 101.2 & 1.2 & 101.5 & 5.2 & 94.1 & 9.5 & 88.4 & 8.1 \\
\hline
\end{tabular}

The 11 Gelsemium alkaloids are basic nitrogen-containing compounds existing in organisms. They have complex nitrogen-containing heterocycles, optical specificity, and significant physiological effects. This study has elucidated the toxicokinetic parameters of 11 Gelsemium alkaloids in rats. After intravenous administration, the concentrations in plasma at different time points were measured, and the toxicokinetic parameters were calculated. The half-lives of rankinidine and humaniline were the shortest, and gelsevirine was the longest, suggesting that rankinidine and humaniline absorbed and eliminated faster, had a short time to reach the peak, and had high plasma concentration. On the other hand, gelsevirine eliminated more slowly, indicating that the maintenance time of efficacy was longer. It also suggests that gelsevirine may accumulate in the body if taken for a long time [40-42]. The $\mathrm{AUC}_{(0-t)}$ values of humantenirine and gelsevirine were higher than the other nine alkaloids, at $73.1 \pm 19.5 \mathrm{ng} /$ $\mathrm{mL} * \mathrm{~h}$ and $73.8 \pm 12.9 \mathrm{ng} / \mathrm{mL} * \mathrm{~h}$, respectively. Conversely, $\mathrm{AUC}_{(0-t)}$ of rankinidine and gelsemine were lower than that of the other nine alkaloids, at $2.2 \pm 0.8 \mathrm{ng} / \mathrm{mL} * \mathrm{~h}$ and $1.7 \pm 0.5 \mathrm{ng} / \mathrm{mL} * \mathrm{~h}$, respectively. The difference in the absorption of the different alkaloids was obvious.

\section{Conclusion}

The UPLC-MS/MS method has been validated for the simultaneous determination of 11 Gelsemium alkaloids in rat plasma, and it was successfully applied in 
TABLE 5: Toxicokinetic parameters of 11 Gelsemium alkaloids after intravenous administration in rats $(0.1 \mathrm{mg} / \mathrm{kg}$ for each alkaloid).

\begin{tabular}{lcccccccc}
\hline Compound & $\begin{array}{c}\mathrm{AUC}_{(0-t)} \\
\mathrm{ng} / \mathrm{mL} * \mathrm{~h}\end{array}$ & $\begin{array}{c}\mathrm{AUC}_{(0-\infty)} \\
\mathrm{ng} / \mathrm{mL} * \mathrm{~h}\end{array}$ & $\begin{array}{c}\mathrm{MRT}_{(0-t)} \\
\mathrm{h}\end{array}$ & $\begin{array}{c}\mathrm{MRT}_{(0-\infty)} \\
\mathrm{h}\end{array}$ & $\begin{array}{c}t_{1 / 2 z} \\
\mathrm{~h}\end{array}$ & $\begin{array}{c}\mathrm{Vz} \\
\mathrm{L} / \mathrm{kg}\end{array}$ & $\begin{array}{c}\mathrm{CL}_{z / F} \\
\mathrm{~L} / \mathrm{h} / \mathrm{kg}\end{array}$ & $\begin{array}{c}C_{\max } \\
\mathrm{ng} / \mathrm{mL}\end{array}$ \\
\hline Humantenirine & $73.1 \pm 19.5$ & $73.2 \pm 19.5$ & $1.4 \pm 0.1$ & $1.4 \pm 0.1$ & $0.9 \pm 0.3$ & $2.0 \pm 0.8$ & $1.4 \pm 0.4$ & $43.7 \pm 6.9$ \\
Humantenine & $14.7 \pm 3.7$ & $14.0 \pm 3.7$ & $1.2 \pm 0.1$ & $1.2 \pm 0.1$ & $1.5 \pm 0.3$ & $15.9 \pm 4.9$ & $7.6 \pm 2.3$ & $11.5 \pm 3.6$ \\
Akuammidine & $4.7 \pm 1.31 .3$ & $4.7 \pm 1.2$ & $1.0 \pm 0.4$ & $1.2 \pm 0.5$ & $2.3 \pm 1.2$ & $84.0 \pm 63.3$ & $22.5 \pm 6.5$ & $7.8 \pm 2.1$ \\
Gelsevirine & $73.8 \pm 12.9$ & $122.7 \pm 107.2$ & $7.2 \pm 1.6$ & $22.0 \pm 31.1$ & $15.8 \pm 8.1$ & $14.2 \pm 6.3$ & $1.2 \pm 0.5$ & $13.2 \pm 3.9$ \\
Rankinidine & $2.2 \pm 0.8$ & $2.2 \pm 0.8$ & $1.0 \pm 0.2$ & $1.1 \pm 0.2$ & $0.9 \pm 0.1$ & $67.5 \pm 22.7$ & $52.7 \pm 22.4$ & $2.3 \pm 1.2$ \\
n-Methoxyanhydrovobasinediol & $3.1 \pm 0.7$ & $3.6 \pm 0.7$ & $1.8 \pm 0.5$ & $3.3 \pm 2.0$ & $2.7 \pm 1.9$ & $106.9 \pm 72.2$ & $28.3 \pm 5.7$ & $2.4 \pm 1.4$ \\
Gelsenicine & $7.5 \pm 4.9$ & $7.6 \pm 4.9$ & $0.8 \pm 0.2$ & $0.8 \pm 0.2$ & $1.4 \pm 1.0$ & $3.2 \pm 2.2$ & $1.7 \pm 0.8$ & $9.4 \pm 4.9$ \\
Gelsemine & $1.7 \pm 0.5$ & $2.0 \pm 0.6$ & $2.1 \pm 0.4$ & $3.7 \pm 2.1$ & $3.0 \pm 1.7$ & $216.2 \pm 104.3$ & $53.0 \pm 16.3$ & $1.1 \pm 0.5$ \\
Koumine & $7.9 \pm 2.3$ & $8.5 \pm 2.7$ & $0.8 \pm 0.2$ & $1.2 \pm 0.5$ & $1.1 \pm 0.6$ & $21.5 \pm 12.6$ & $13.6 \pm 7.3$ & $10.3 \pm 3.6$ \\
Koumidine & $6.7 \pm 2.9$ & $6.9 \pm 2.9$ & $1.8 \pm 0.3$ & $2.1 \pm 0.5$ & $2.2 \pm 1.0$ & $54.4 \pm 34.7$ & $17.1 \pm 7.5$ & $5.4 \pm 2.1$ \\
Sempervirine & $25.7 \pm 6.8$ & $29.4 \pm 9.7$ & $6.5 \pm 2.1$ & $9.7 \pm 4.9$ & $7.7 \pm 3.8$ & $37.2 \pm 12.1$ & $3.7 \pm 1.2$ & $9.3 \pm 4.4$ \\
\hline
\end{tabular}
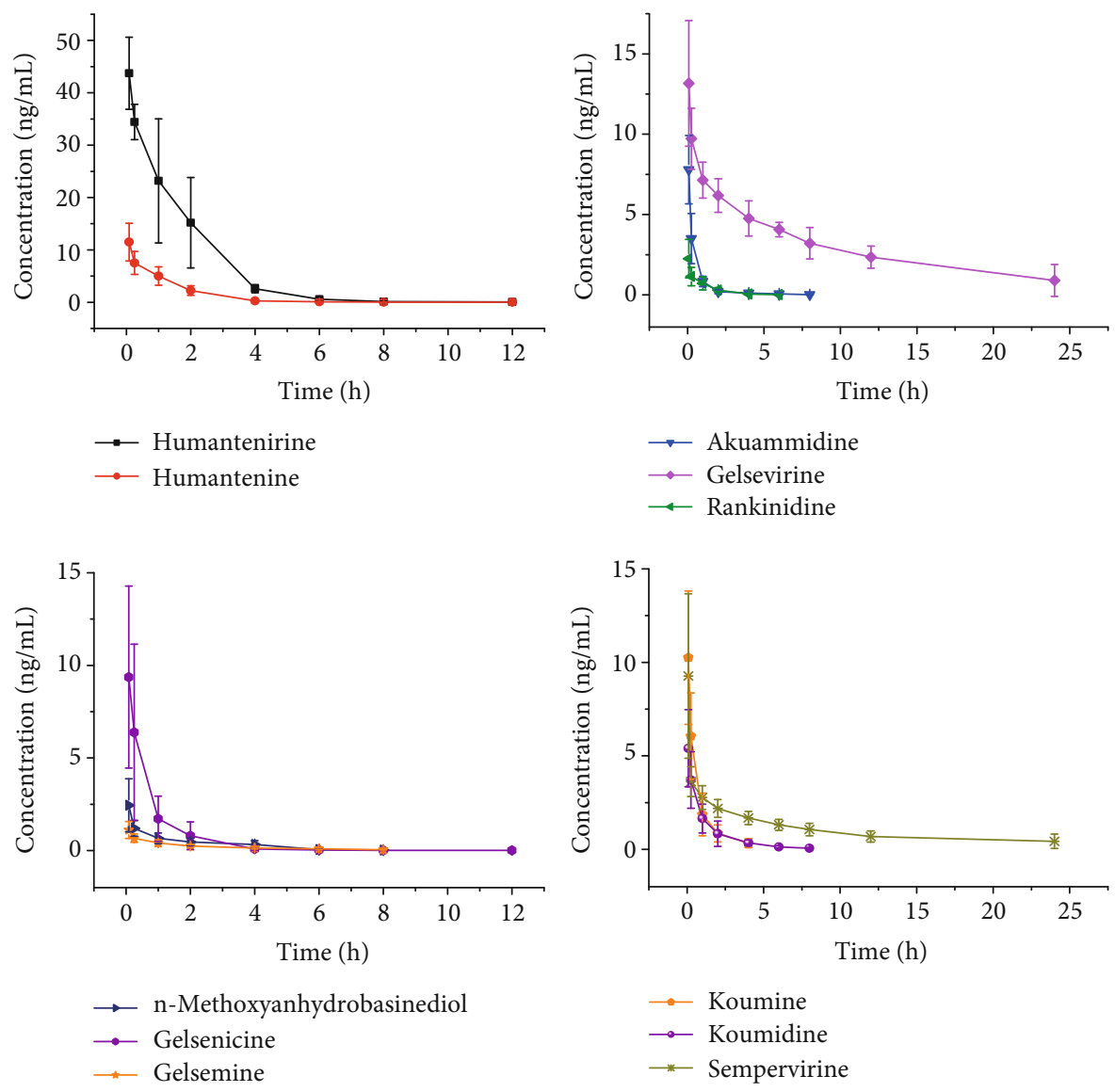

FIGURE 4: The toxicokinetic profiles of humantenirine, humantenine, akuammidine, gelsevirine, rankinidine, n-methoxyanhydrovobasinediol, gelsenicine, gelsemine, koumine, koumidine, and sempervirine in rats $(n=6)$.

toxicokinetic research of 11 Gelsemium alkaloids (humantenirine, humantenine, akuammidine, gelsevirine, rankinidine, n-methoxyanhydrovobasinediol, gelsenicine, gelsemine, koumine, koumidine, and sempervirine) in rats. The toxicoki- netic results provide a basis for the study of the pharmacology and toxicology of Gelsemium alkaloids and provide scientific evidence for the clinical use of $\mathrm{Gel}$ semium alkaloids. 


\section{Data Availability}

The data used to support the findings of this study are included within the article.

\section{Conflicts of Interest}

The authors declare that there is no conflict of interest regarding the publication of this paper.

\section{Acknowledgments}

This study was supported by grants from the Wenzhou Science and Technology Bureau (Y20190661).

\section{References}

[1] Y. Chen, Y. Li, Y. Wang et al., "Comparative pharmacokinetics of active alkaloids after oral administration of Rhizoma Coptidis extract and Wuji Wan formulas in rat using a UPLCMS/MS method," European Journal of Drug Metabolism and Pharmacokinetics, vol. 40, no. 1, pp. 67-74, 2015.

[2] E. Chevlen, "Morphine with dextromethorphan: conversion from other opioid analgesics," Journal of Pain and Symptom Management, vol. 19, 1 Supplement, pp. S42-S49, 2000.

[3] S. Wang, Y. Dong, K. Su et al., "Effect of codeine on CYP450 isoform activity of rats," Pharmaceutical Biology, vol. 55, no. 1, pp. 1223-1227, 2017.

[4] J. X. Zhai, M. Shen, and W. Liu, "Simultaneous screening for 45 poisonous alkaloids in blood by LC-MS/MS," Fa Yi Xue Za Zhi, vol. 31, no. 1, pp. 28-33, 2015.

[5] Y. Liu, Q. Tang, P. Cheng et al., "Whole-genome sequencing and analysis of the Chinese herbal plant Gelsemium elegans," Acta Pharmaceutica Sinica B, vol. 10, no. 2, pp. 374-382, 2020.

[6] T. Y. A. Chow, C. H. V. Ng, and M. L. Tse, "Clinical manifestations and causes of gelsemium poisoning in Hong Kong from 2005 to 2017: review of 33 cases," Hong Kong Journal of Emergency Medicine, vol. 26, no. 6, pp. 351-356, 2019.

[7] L. Fang, J. Zhou, Y. L. Lin et al., "Large-scale separation of alkaloids from Gelsemium elegans by $\mathrm{pH}$-zone- refining countercurrent chromatography with a new solvent system screening method," Journal of Chromatography A, vol. 1307, pp. 80-85, 2013.

[8] M. Kitajima, H. Kobayashi, N. Kogure, and H. Takayama, "New oxindole and indole alkaloids from Gelsemium rankinii," Tetrahedron, vol. 66, no. 32, pp. 5987-5992, 2010.

[9] B. O. Johnson, A. M. Golonka, A. Blackwell, I. Vazquez, and N. Wolfram, "Floral scent variation in the heterostylous species Gelsemium sempervirens," Molecules, vol. 24, no. 15, p. $2818,2019$.

[10] G. L. Jin, Y. P. Su, M. Liu et al., "Medicinal plants of the genus Gelsemium (Gelsemiaceae, Gentianales) -a review of their phytochemistry, pharmacology, toxicology and traditional use," Journal of Ethnopharmacology, vol. 152, no. 1, pp. 33$52,2014$.

[11] L. Sun, F. Ding, G. You et al., "Development and validation of an UPLC-MS/MS method for pharmacokinetic comparison of five alkaloids from Jin Qi Jiangtang tablets and its monarch drug Coptidis Rhizoma," Pharmaceutics, vol. 10, no. 1, 2018.

[12] Y. Tao, S. Huang, J. Yan, and B. Cai, "Establishment of a rapid and sensitive UPLC-MS/MS method for pharmacokinetic determination of nine alkaloids of crude and processed Corydalis turtschaninovii Besser aqueous extracts in rat plasma," Journal of Chromatography. B, Analytical Technologies in the Biomedical and Life Sciences, vol. 1124, pp. 218-225, 2019.

[13] J. Wei, L. Fang, X. Liang, D. Su, and X. Guo, "A sensitive and selective UPLC-MS/MS method for simultaneous determination of 10 alkaloids from Rhizoma Menispermi in rat plasma and its application to a pharmacokinetic study," Talanta, vol. 144, pp. 662-670, 2015.

[14] J. Wei, Z. Jiang, Z. Cui, and X. Guo, "Rapid determination of eight oxoisoaporphine alkaloids in Rhizoma Menispermi by the optimal homogenate extraction followed by UPLCMS/MS," Analytical and Bioanalytical Chemistry, vol. 407, no. 18, pp. 5535-5540, 2015.

[15] H. Qiu, C. Yu, Y. Cheng et al., "Simultaneous determination of koumine and gelsemine in human plasma using HPLC-UV assay and its clinical application," Current Pharmaceutical Analysis, vol. 15, no. 6, pp. 640-649, 2019.

[16] S.-S. Liu, K. Yang, Z.-L. Sun, X. Zheng, X. Bai, and Z.-Y. Liu, "A novel two-dimensional liquid chromatography system for the simultaneous determination of three monoterpene indole alkaloids in biological matrices," Analytical and Bioanalytical Chemistry, vol. 411, no. 17, pp. 3857-3870, 2019.

[17] M.-T. Zuo, Z.-Y. Wang, K. Yang et al., "Characterization of absorbed and produced constituents in goat plasma urine and faeces from the herbal medicine Gelsemium elegans by using high- performance liquid chromatography coupled with quadrupole time-of-flight mass spectrometry," Journal of Ethnopharmacology, vol. 252, pp. 112617-112617, 2020.

[18] K. Yang, X.-M. Long, J.-J. Cao et al., “An analytical strategy to explore the multicomponent pharmacokinetics of herbal medicine independently of standards: Application in Gelsemium elegans extracts," Journal of Pharmaceutical and Biomedical Analysis, vol. 176, p. 112833, 2019.

[19] K. Yang, X.-M. Long, Y.-C. Liu et al., "Development and inhouse validation of a sensitive LC-MS/MS method for simultaneous quantification of gelsemine, koumine and humantenmine in porcine plasma," Journal of Chromatography B-Analytical Technologies in the Biomedical and Life Sciences, vol. 1076, pp. 54-60, 2018.

[20] Y. Hu, M. Chen, Z. Wang et al., "Development of a validated UPLC-MS/MS method for determination of humantenmine in rat plasma and its application in pharmacokinetics and bioavailability studies," Biomedical Chromatography, vol. 31, no. 12, 2017.

[21] S. Zhang, S. Hu, X. Yang et al., "Development of a liquid chromatography with mass spectrometry method for the determination of gelsemine in rat plasma and tissue: application to a pharmacokinetic and tissue distribution study," Journal of Separation Science, vol. 38, no. 6, pp. 936-942, 2015.

[22] S. Tong, Z. Wang, S. Jiang, and J. Cai, "Determination of gelsemine in rat plasma by liquid chromatography mass spectrometry and its application to a pharmacokinetic study," Latin American Journal of Pharmacy, vol. 33, no. 6, pp. 988-993, 2014.

[23] X. Wu, W. Huang, L. Lu, L. Lin, and X. Yang, "Simultaneous determination of six alkaloids in blood and urine using a hydrophilic interaction liquid chromatography method coupled with electrospray ionization tandem mass spectrometry," Analytical and Bioanalytical Chemistry, vol. 398, no. 3, pp. 1319-1327, 2010.

[24] J.-J. Cao, K. Yang, C.-Y. Huang et al., "Pharmacokinetic study of multiple components of Gelsemium elegans in goats by 
ultra-performance liquid chromatography coupled to tandem mass spectrometry," Journal of Analytical Toxicology, vol. 44, no. 4, pp. 378-390, 2020.

[25] J. Li, Y. Jin, H. Fu, Y. Huang, X. Wang, and Y. Zhou, "Pharmacokinetics and bioavailability of gelsenicine in mice by UPLCMS/MS," Biomedical Chromatography, vol. 33, no. 3, p. e4418, 2019.

[26] L. Wang, Y. Wen, and F. Meng, "Simultaneous determination of gelsemine and koumine in rat plasma by UPLC-MS/MS and application to pharmacokinetic study after oral administration of Gelsemium elegans Benth extract," Biomedical Chromatography, vol. 32, no. 6, p. e4201, 2018.

[27] L. Wang, Q. Sun, N. Zhao, Y.-Q. Wen, Y. Song, and F.-H. Meng, "Ultra-liquid chromatography tandem mass spectrometry (UPLC-MS/MS)-based pharmacokinetics and tissue distribution study of koumine and the detoxification mechanism of Glycyrrhiza uralensis Fisch on Gelsemium elegans Benth," Molecules, vol. 23, no. 7, p. 1693, 2018.

[28] J.-Z. Chen, Y. Li, J.-P. Xiao, S.-S. Wu, and H.-W. Song, "Development of a sensitive and rapid UPLC-MS/MS method for the determination of koumine in rat plasma: application to a pharmacokinetic study," Biomedical Chromatography, vol. 27, no. 6, pp. 736-740, 2013.

[29] K. Liu, D. Zhong, and X. Chen, "Enantioselective determination of doxazosin in human plasma by liquid chromatographytandem mass spectrometry using ovomucoid chiral stationary phase," Journal of Chromatography B-Analytical Technologies in the Biomedical and Life Sciences, vol. 878, no. 26, pp. 24152420, 2010.

[30] S. Y. Son, H. S. Rhee, M. W. Lee, and J. M. Park, "Analysis of benzo[c]phenanthridine alkaloids inEschscholtzia californicacell culture using HPLC-DAD and HPLC-ESI-MS/MS," Bioscience, Biotechnology, and Biochemistry, vol. 78, no. 7, pp. 1103-1111, 2014.

[31] W. Kukula-Koch and T. Mroczek, "Application of hydrostatic CCC-TLC-HPLC-ESI-TOF-MS for the bioguided fractionation of anticholinesterase alkaloids from Argemone mexicana L. roots," Analytical and Bioanalytical Chemistry, vol. 407, no. 9, pp. 2581-2589, 2015.

[32] J. Liu, Q. Li, Y. Yin, R. Liu, H. Xu, and K. Bi, "Ultra-fast LC-ESI-MS/MS method for the simultaneous determination of six highly toxic Aconitum alkaloids from Aconiti kusnezoffii radix in rat plasma and its application to a pharmacokinetic study," Journal of Separation Science, vol. 37, no. 1-2, pp. 171-178, 2014.

[33] C. Wen, J. Cai, C. Lin, J. Ma, and X. Wang, "Gradient elution liquid chromatography mass spectrometry determination of acetylcorynoline in rat plasma and its application to a pharmacokinetic study," Xenobiotica, vol. 44, no. 8, pp. 743-748, 2014.

[34] J. Ma, X. Ding, C. Sun et al., "Development and validation a liquid chromatography mass spectrometry for determination of solasodine in rat plasma and its application to a pharmacokinetic study," Journal of Chromatography. B, Analytical Technologies in the Biomedical and Life Sciences, vol. 963, pp. 24-28, 2014.

[35] D. Dabrowski, K. Lech, and M. Jarosz, "Capillary-HPLC with tandem mass spectrometry in analysis of alkaloid dyestuffs a new approach," Electrophoresis, vol. 39, no. 9-10, pp. 12761283, 2018.

[36] A. Petruczynik, T. Tuzimski, T. Plech, J. Misiurek, K. Szalast, and G. Szymczak, "Comparison of anticancer activity and HPLC-DAD determination of selected isoquinoline alkaloids from Thalictrum foetidum, Berberis sp. and Chelidonium majus extracts," Molecules, vol. 24, no. 19, p. 3417, 2019.

[37] K. Nesmerak, K. Kudlacek, M. Sticha, V. Cerveny, J. Kunesova, and I. Yildiz, "HPLC-MS analysis of ipecacuanha alkaloids in pharmaceutical relics from eighteenth century," Monatshefte Fur Chemie, vol. 149, no. 9, pp. 1535-1542, 2018.

[38] X. Y. Cheng, Y. Shi, S. L. Zhen, H. Sun, and W. Jin, "HPLC-MS analysis of ethanol extract of Corydalis yanhusuo and simultaneous determination of eight protoberberine quaternary alkaloids by HPLC-DAD," Journal of Chromatographic Science, vol. 48, no. 6, pp. 441-444, 2010.

[39] S. Zou, Y. Ge, X. Chen et al., "Simultaneous determination of five alkaloids by HPLC-MS/MS combined with micro-SPE in rat plasma and its application to pharmacokinetics after oral administration of lotus leaf extract," Frontiers in Pharmacology, vol. 10, p. 1252, 2019.

[40] M. S. Sisti, A. Scilingo, and M. C. Anon, "Effect of the incorporation of amaranth (Amaranthus mantegazzianus) into fatand cholesterol-rich diets for Wistar rats," Journal of Food Science, vol. 84, no. 11, pp. 3075-3082, 2019.

[41] S. Cang, R. Liu, T. Wang et al., "Simultaneous determination of five active alkaloids from Compound Kushen Injection in rat plasma by LC-MS/MS and its application to a comparative pharmacokinetic study in normal and NSCLC nude rats," Journal of Chromatography. B, Analytical Technologies in the Biomedical and Life Sciences, vol. 1126-1127, p. 121734, 2019.

[42] N. Chen, C.-e. Guo, H. Chen et al., "Simultaneous determination of six coptis alkaloids in urine and feces by LC-MS/MS and its application to excretion kinetics and the compatibility mechanism of Jiao-Tai-Wan in insomniac rats," Biomedical Chromatography, vol. 32, no. 8, p. e4248, 2018. 